\title{
Mobilizing Animal Movement Data: API use and the Movebank platform
}

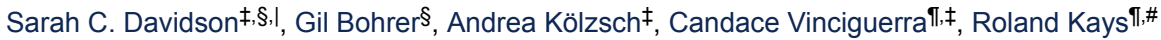 \\ ‡ Max Planck Institute of Animal Behavior, Radolfzell, Germany \\ $\S$ The Ohio State University, Columbus, United States of America \\ | University of Konstanz, Konstanz, Germany \\ II North Carolina Museum of Natural Sciences, Raleigh, United States of America \\ \# North Carolina State University, Forestry and Environmental Resources, Raleigh, United States of America
}

Corresponding author: Sarah C. Davidson (sdavidson@ab.mpg.de)

Received: 11 Sep 2021 | Published: 13 Sep 2021

Citation: Davidson SC, Bohrer G, Kölzsch A, Vinciguerra C, Kays R (2021) Mobilizing Animal Movement Data:

API use and the Movebank platform. Biodiversity Information Science and Standards 5: e74312.

https://doi.org/10.3897/biss.5.74312

\begin{abstract}
Movebank, a global platform for animal tracking and other animal-borne sensor data, is used by over 3,000 researchers globally to harmonize, archive and share nearly 3 billion animal occurrence records and more than 3 billion other animal-borne sensor measurements that document the movements and behavior of over 1,000 species. Movebank's publicly described data model (Kranstauber et al. 2011), vocabulary and application programming interfaces (APIs) provide services for users to automate data import and retrieval. Near-live data feeds are maintained in cooperation with over 20 manufacturers of animal-borne sensors, who provide data in agreed-upon formats for accurate data import. Data acquisition by API complies with public or controlled-access sharing settings, defined within the database by data owners. The Environmental Data Automated Track Annotation System (EnvDATA, Dodge et al. 2013) allows users to link animal tracking data with hundreds of environmental parameters from remote sensing and weather reanalysis products through the Movebank website, and offers an API for advanced users to automate the submission of annotation requests. Movebank's mobile apps, the Animal Tracker and Animal Tagger, use APIs to support reporting and monitoring while in the field, as well as communication with citizen scientists. The recently-launched MoveApps platform connects with Movebank data using an API to allow users to build,
\end{abstract}


execute and share repeatable workflows for data exploration and analysis through a userfriendly interface. A new API, currently under development, will allow calls to retrieve data from Movebank reduced according to criteria defined by "reduction profiles", which can greatly reduce the volume of data transferred for many use cases.

In addition to making this core set of Movebank services possible, Movebank's APIs enable the development of external applications, including the widely used $\mathrm{R}$ programming packages 'move' (Kranstauber et al. 2012) and 'ctmm' (Calabrese et al. 2016), and userspecific workflows to efficiently execute collaborative analyses and automate tasks such as syncing with local organizational and governmental websites and archives. The APIs also support large-scale data acquisition, including for projects under development to visualize, map and analyze bird migrations led by the British Trust for Ornithology, the coordinating organisation for European bird ringing (banding) schemes (EURING), Georgetown University, National Audubon Society, Smithsonian Institution and United Nations Convention on Migratory Species.

Our API development is constrained by a lack of standardization in data reporting across animal-borne sensors and a need to ensure adequate communication with data users (e.g., how to properly interpret data; expectations for use and attribution) and data owners (e.g., who is using publicly-available data and how) when allowing automated data access. As interest in data linking, harvesting, mirroring and integration grows, we recognize needs to coordinate API development across animal tracking and biodiversity databases, and to develop a shared system for unique organism identifiers. Such a system would allow linking of information about individual animals within and across repositories and publications in order to recognize data for the same individuals across platforms, retain provenance and attribution information, and ensure beneficial and biologically meaningful data use.

\section{Keywords}

bio-logging, movement ecology, real-time data, software development

\section{Presenting author}

Sarah C. Davidson

\section{Presented at}

TDWG 2021 


\section{References}

- Calabrese J, Fleming C, Gurarie E (2016) ctmm: an R package for analyzing animal relocation data as a continuous-time stochastic process. Methods in Ecology and Evolution 7 (9): 1124-1132. https://doi.org/10.1111/2041-210x.12559

- Dodge S, Bohrer G, Weinzierl R, Davidson SC, Kays R, Douglas D, Cruz S, Han J, Brandes D, Wikelski M (2013) The environmental-data automated track annotation (Env-DATA) system: linking animal tracks with environmental data. Movement Ecology 1 https://doi.org/10.1186/2051-3933-1-3

- Kranstauber B, Cameron A, Weinzierl R, Fountain T, Tilak S, Wikelski M, Kays R, et al. (2011) The Movebank data model for animal tracking. Environ Model Softw 26 (6): 834-835. https://doi.org/10.1016/j.envsoft.2010.12.005

- Kranstauber B, Kays R, LaPoint S, Wikelski M, Safi K (2012) A dynamic Brownian bridge movement model to estimate utilization distributions for heterogeneous animal movement. Journal of Animal Ecology 81 (4): 738-746. https://doi.org/10.1111/j. 1365-2656.2012.01955.x 